\title{
Poster Abstract: Towards a Heterogeneous Internet-of-Things Testbed via Mesh inside a Mesh
}

\author{
Luwen Miao, Kaikai Liu \\ Department of Computer Engineering \\ San Jose State University \\ San Jose, CA, USA \\ \{luwen.miao,kaikai.liu\}@sjsu.edu
}

\begin{abstract}
Connectivity is at the heart of the future Internet-of-Things (IoT) infrastructure, which can control and communicate with remote sensors and actuators for the beacons, data collections, and forwarding nodes. Existing sensor network solutions cannot solve the bottleneck problems near the sink node; the tree-based Internet architecture has the single point of failure. To solve current deficiencies in multi-hop mesh network and cross-domain network design, we propose a mesh inside a mesh IoT network architecture. Our designed "edge router" incorporates these two mesh networks together and performs seamlessly transmission of multi-standard packets. The proposed IoT testbed interoperates with existing multi-standards (Wi-Fi, 6LoWPAN) and segments of networks, and provides both high-throughput Internet and resilient sensor coverage throughout the community.
\end{abstract}

\section{INTRODUCTION}

The use of sensors and Internet-of-Things (IoT) to capture and transmit data that can help a city operate in an intelligent and sustainable way has become widespread and is gaining tremendous attention and popularity worldwide.

Existing Internet and sensor network infrastructure has one significant drawback: interoperability. Existing Sensor network leverages mesh network connection, and delivers the data to the sink node (gateway) through multiple hops. However, the nodes close to the sink have significantly shorter battery life (shorter overall network life time) due to frequent data forwarding; the bandwidth close to the sink often becomes the bottleneck [2]. Beyond the sink node, existing sensor network solutions cannot cover the data transmission outside the boundary, for example, sending through the Internet via $\mathrm{Wi}-\mathrm{Fi}$ or Ethernet. IETF is standardizing the 6LoWPAN, RPL and CoAP as the network and application layer protocol for low-powered and lossy networked environ-

\footnotetext{
* The work presented in this paper is supported in part by National Science Foundation under Grant No. CNS1637371 .
}

\footnotetext{
Permission to make digital or hard copies of part or all of this work for personal or classroom use is granted without fee provided that copies are not made or distributed for profit or commercial advantage and that copies bear this notice and the full citation on the first page. Copyrights for third-party components of this work must be honored. For all other uses, contact the owner/author(s).

SenSys '16 November 14-16, 2016, Stanford, CA, USA

(C) 2016 Copyright held by the owner/author(s).

ACM ISBN 978-1-4503-4263-6/16/11 . \$ \$15.00

DOI: http://dx.doi.org/10.1145/2994551.2996710
}

ments [1], and try to unify the sensor network address world with the Internet world via IPv6. Other than IETF, many companies have developed their own systems and protocols that are meant specifically for IoT systems. For example, The building automation system consists of fire detectors, smoke alarms, surveillance cameras, entertainment systems, and lighting systems. All of the nodes have to be interoperable. Existing systems, for example, Contiki, Google's Brillo, Apple's Homekit, still works in a simple domain, i.e., achieving the interoperability via a single communication protocol like Zigbee, BLE or WiFi [3]. When nodes are deployed in thousands with sparse link connections, how can they be efficiently connected and managed within budget? Many real world IoT applications have more complicated deployment environments. For example, the sink node is still far away from the Internet; the bandwidth is insufficient near the sink when only using the low complex protocol; existing Wi-Fi infrastructure cannot be utilized.

\section{SYSTEM DESIGN}

To solve current deficiencies of the interoperability problem, we propose a mesh inside a mesh IoT network architecture. The first mesh is the sensor network mesh powered by the 6LoWPAN and IETF RPL. We utilize the TI SensorTag 2 as the node of the sensor mesh. The SensorTag equipped with ten sensors, and the communication protocol can be multi-standard (Zigbee, 6LoWPAN, and BLE) by just changing the firmware.

The second mesh is the low cost Wi-Fi mesh network powered by the HSMM-Pi. Instead of deploying a traditional Wi-Fi infrastructure with 2-layers (Ethernet cable connected to all Wi-Fi nodes), we utilize low complex mesh Wi-Fi network to supplement the limited cable coverage. We leverage the low cost Raspberry Pi 3 as the Wi-Fi mesh router via the HSMM-Pi. In the mean time, this "edge router" also connects the 6LoWPAN mesh (the sensor mesh) by plugging a Zigbee dongle to the Raspberry Pi 3. The "edge router" consists the $6 \mathrm{LBR}$ on Raspberry $\mathrm{Pi} 3$, and SLIP radio for the dongle.

\section{PRELIMINARY RESULTS}

Experimental Setup. To evaluate the key performance metrics, we established a minimal mesh network testbed as shown in Fig. 1. This testbed consists of heterogeneous devices: 1) one Raspberry Pi 2 with CanaKit Wi-Fi adapter; 2) one Raspberry Pi 3; 3) one Linksys EA3500 router and Linksys WRT1900ACS router. 


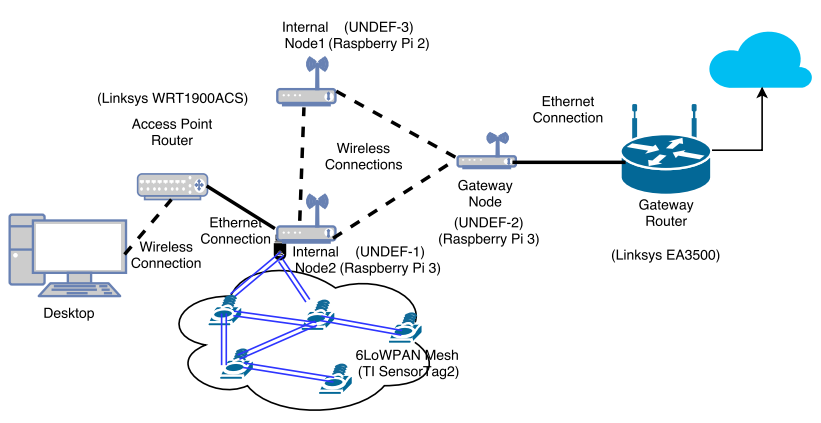

Figure 1: Experimental Architecture.

Performance Metrics. To evaluate our design, we introduced benchmarks to test the performance of the proposed mesh inside a mesh network. We tested three main metrics: throughput in terms of TCP and UDP performance, network coverage, and the data forwarding function. The mesh network works as the infrastructure for the connected device and responsible for forwarding the data. We tested two types of data throughput: the internal mesh throughput and the throughput between the connected mobile device and the Internet. We use iPerf as the network bandwidth measurement tool.

Throughput between internal node and gateway node: We manually run the iPerf bandwidth tests 10 times for TCP transmission and UDP transmission, and check the highest and lowest data throughput. We calculate the mean average of data throughput using these 10 times' measured data. The results are showed in Fig. 2. From Fig. 2a, the highest data rate in TCP transmission is $13.2 \mathrm{Mbits} / \mathrm{sec}$ for the gateway node and $13.7 \mathrm{Mbits} / \mathrm{sec}$ for the internal node. From Fig. 2b, the data rate in UDP transmission is stable and the value is $1.05 \mathrm{Mbits} / \mathrm{sec}$ for both the gateway node and the internal node. The data lost ratio is as low as $0.11 \%$.

Throughput between internal nodes: The mesh network contains multiple internal nodes. In order to fairly test the performance of internal throughput, we perform multiple server/client settings for the iPerf measurement. From Fig. 2a, the TCP throughput value is around 6.16 Mbits/sec. The data rate under UDP connection is steady $1.05 \mathrm{Mbits} / \mathrm{sec}$ in Fig. 2b. The data lost ratio is 0\%. Compared with the throughput between internal node and gateway node, the value is lower. This is caused by the lower performance of the internal node, i.e., Raspberry Pi.

Throughput between the mobile device and the Internet: In order to test the service data rate (connect to the Internet) of the mobile device, we use iPerf3 server as the testing server. We specifically use the iperf.he.net that is located at Fremont, CA. From Fig. 2a, the highest TCP data rate achieved is $579 \mathrm{Kbits} / \mathrm{sec}$; the average data rate is $285.33 \mathrm{Kbits} / \mathrm{sec}$. The UDP data rate is stable, it is still around 1.05 Mbits/sec as shown in Fig. 2b.

Mesh Network Coverage: We measure the TCP and UDP data rate and UDP data lost ratio for 1 minute every 10 meters. The measure interval is 6 seconds for every 1 minute.

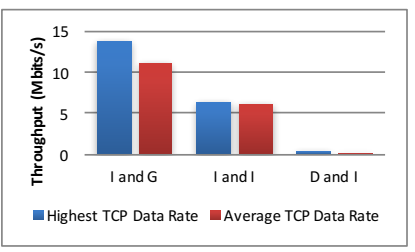

(a) TCP Throughput

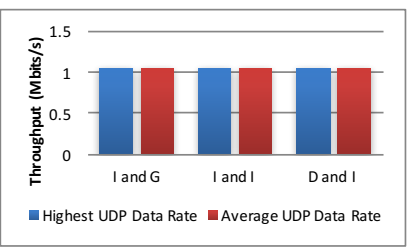

(b) UDP Throughput
Figure 2: 1) TCP throughput and 2) UDP throughput of the mesh network. I and $\mathrm{G}$ means data rate between internal node and gateway node. I and I means data rate between internal nodes. D and I means data rate between connected device and the Internet.

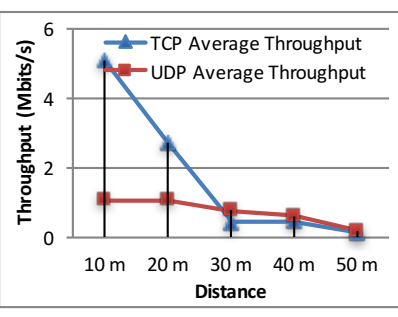

(a) Throughput vs. distance

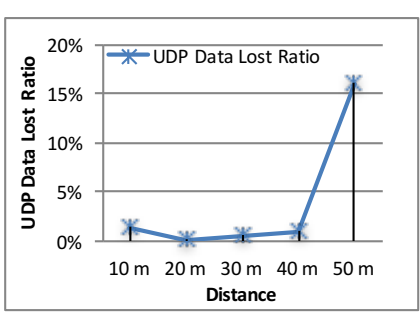

(b) Data lost ratio vs. distance
Figure 3: 1) TCP and UDP throughput vs. distance between internal mesh nodes; 2) UDP data lost ratio vs. distance.

As shown in Fig. 3a, the TCP data rate is significantly decreased when the distance increases, the UDP throughput is decreased form 1.05 Mbits/sec to $172 \mathrm{Kbits} / \mathrm{sec}$. The UDP data lost ratio is increasing as shown in Fig. $3 \mathrm{~b}$.

\section{CONCLUSION}

Our preliminary system demo can be access via this link: https://youtu.be/tNAvqO5hW5M. Our future works includes add more node (both Wi-Fi and Sensors), improves our solutions in terms of system setup time and resilient. We will also test the large scale networking performance with real sensor data in a truly hybrid mesh situations.

\section{REFERENCES}

[1] J. Hui, J. Vasseur, D. Culler, and V. Manral. An ipv6 routing header for source routes with the routing protocol for low-power and lossy networks (rpl). Technical report, 2012.

[2] Y. Liu, Y. He, M. Li, J. Wang, K. Liu, and X. Li. Does wireless sensor network scale? a measurement study on greenorbs. IEEE Transactions on Parallel and Distributed Systems, 24(10):1983-1993, 2013.

[3] V. Sevani, B. Raman, and P. Joshi. Implementation-based evaluation of a full-fledged multihop tdma-mac for wifi mesh networks. IEEE Transactions on Mobile Computing, 13(2):392-406, 2014. 